\title{
Losartan Prevents Maladaptive Auditory-Somatosensory Plasticity After Hearing Loss via Transforming Growth Factor- $\beta$ Signaling Suppression
}

\author{
Seog-Kyun Mun ${ }^{1} \cdot$ Kyu-Hee Han² $\cdot$ Jong Tae Baek ${ }^{2}$ Suk-Won Ahn ${ }^{3} \cdot$ Hyun Sang Cho ${ }^{4}$ Mun Young Chang ${ }^{1,5}$ \\ ${ }^{1}$ Department of Otorhinolaryngology-Head and Neck Surgery, Chung-Ang University College of Medicine, Seoul; \\ ${ }^{2}$ Department of Otorhinolaryngology, National Medical Center, Seoul; \\ ${ }^{3}$ Department of Neurology, Chung-Ang University College of Medicine, Seoul; \\ ${ }^{4}$ Department of Otorhinolaryngology-Head and Neck Surgery, Veterans Health Service Medical Center, Seoul; \\ ${ }^{5}$ Biomedical Research Institute, Chung-Ang University Hospital, Seoul, Korea
}

Objectives. Hearing loss disrupts the balance of auditory-somatosensory inputs in the cochlear nucleus (CN) of the brainstem, which has been suggested to be a mechanism of tinnitus. This disruption results from maladaptive auditory-somatosensory plasticity, which is a form of axonal sprouting. Axonal sprouting is promoted by transforming growth factor (TGF)- $\beta$ signaling, which can be inhibited by losartan. We investigated whether losartan prevents maladaptive auditory-somatosensory plasticity after hearing loss.

Methods. The study consisted of two stages: determining the time course of auditory-somatosensory plasticity following hearing loss and preventing auditory-somatosensory plasticity using losartan. In the first stage, rats were randomly divided into two groups: a control group that underwent a sham operation and a deaf group that underwent cochlea ablation on the left side. CNs were harvested 1 and 2 weeks after surgery. In the second stage, rats were randomly divided into either a saline group that underwent cochlear ablation on the left side and received normal saline or a losartan group that underwent cochlear ablation on the left side and received losartan. CNs were harvested 2 weeks after surgery. Hearing was estimated with auditory brainstem responses (ABRs). Western blotting was performed for vesicular glutamate transporter 1 (VGLUT1), reflecting auditory input; vesicular glutamate transporter 2 (VGLUT2), reflecting somatosensory input; growth-associated protein 43 (GAP-43), reflecting axonal sprouting; and p-Smad2/3.

Results. Baseline ABR thresholds before surgery ranged from 20 to $35 \mathrm{~dB}$ sound pressure level. After cochlear ablation, ABR thresholds were higher than $80 \mathrm{~dB}$. In the first experiment, VGLUT2/VGLUT1 ratios did not differ significantly between the control and deaf groups 1 week after surgery. At 2 weeks after surgery, the deaf group had a significantly higher VGLUT2/VGLUT1 ratio compared to the control group. In the second experiment, the losartan group had a significantly lower VGLUT2/VGLUT1 ratio along with significantly lower p-Smad3 and GAP-43 levels compared to the saline group.

Conclusion. Losartan might prevent axonal sprouting after hearing loss by blocking TGF- $\beta$ signaling thereby preventing maladaptive auditory-somatosensory plasticity.

Keywords. Hearing Loss; Auditory-Somatosensory Plasticity; Tinnitus; Losartan

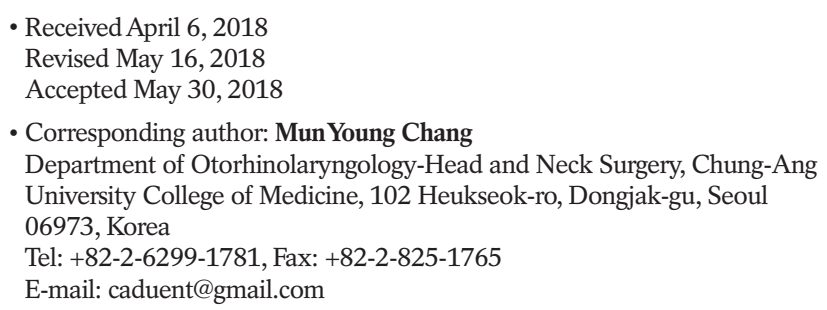

\section{INTRODUCTION}

The cochlear nucleus of the brainstem is the first site of the auditory system where auditory and somatosensory inputs converge [1]. Somatosensory inputs originate from the dorsal root ganglion, trigeminal ganglion, spinal trigeminal nucleus and dorsal column nucleus [2,3]. Hearing loss has been reported to

Copyright () 2019 by Korean Society of Otorhinolaryngology-Head and Neck Surgery.

This is an open-access article distributed under the terms of the Creative Commons Attribution Non-Commercial License (http://creativecommons.org/licenses/by-nc/4.0)

which permits unrestricted non-commercial use, distribution, and reproduction in any medium, provided the original work is properly cited. 
cause cross-modal compensation in the cochlear nucleus, which has been suggested as one of the mechanisms of tinnitus [4,5]. The main results of cross-modal compensation are a disrupted balance of auditory and somatosensory inputs, which has been demonstrated by measuring changes in vesicular glutamate transporter 1 (VGLUT1) and vesicular glutamate transporter 2 (VGLUT2) levels after hearing loss [6,7]. Auditory inputs are exclusively associated with VGLUT1 $[6,8]$ and somatosensory inputs are primarily associated with VGLUT2 [9]. The VGLUT2/ VGLUT1 ratio increases in the dorsal cochlear nucleus after hearing loss due to the decreased auditory input from hearing loss and a subsequent increase in somatosensory input $[6,7]$. This phenomenon is the result of maladaptive auditory-somatosensory plasticity, which is a form of axonal sprouting $[6,10]$.

To date, several studies have provided evidence that maladaptive auditory-somatosensory plasticity in the cochlear nucleus after hearing loss is an important mechanism for tinnitus [4-6], but prevention of this maladaptive auditory-somatosensory plasticity has not been examined. In this study, we attempted to prevent maladaptive auditory-somatosensory plasticity in the cochlear nucleus after hearing loss. First, we investigated the time course of auditory-somatosensory plasticity following hearing loss to determine when the balance of auditory and somatosensory inputs was disrupted. Second, we attempted to prevent maladaptive auditory-somatosensory plasticity with losartan, a clinically relevant transforming growth factor- $\beta$ (TGF- $\beta$ ) inhibitor because TGF- $\beta$ signaling is important in promoting axonal sprouting. The results of this study are expected to contribute to identifying the mechanisms of auditory-somatosensory plasticity and tinnitus.

\section{MATERIALS AND METHODS}

\section{Experimental design}

This study was approved by the Institutional Animal Care and Use Committee of Chung-Ang University (2016-00092). All procedures were performed in accordance with guidelines provided by the Institutional Animal Care and Use Committee of Chung-Ang University. Seven-week-old male Sprague Dawley rats (200-250 g) were used. The study consisted of two stages: determining the time course of auditory-somatosensory plastici-

\section{H I G G H L L I}

- Auditory-somatosensory plasticity occurred 2 weeks after hearing loss.

- Losartan prevented auditory-somatosensory plasticity after hearing loss.

- Losartan might prevent axonal sprouting after hearing loss by blocking transforming growth factor- $\beta$ signaling. ty following hearing loss and preventing auditory-somatosensory plasticity with losartan. In the first stage, 28 rats were randomly divided into two groups: a control group $(n=14)$ that underwent a sham operation and a deaf group $(n=14)$ that underwent unilateral cochlear ablation on the left side. Seven rats in each group were euthanized 1 week after surgery and the left cochlear nuclei were harvested. The remaining seven rats in each group were euthanized 2 weeks after surgery and the left cochlear nuclei were harvested. The results of the first experiment were used to determine the time point when the balance of auditory and somatosensory inputs was disrupted. In the second stage, 14 rats were randomly divided into two experimental groups: a saline group $(n=7)$ that underwent unilateral cochlear ablation on the left side and received normal saline intraperitoneally for 2 weeks and a losartan group $(n=7)$ that underwent unilateral cochlear ablation and received losartan $(100 \mathrm{mg} / \mathrm{kg})$ intraperitoneally for 2 weeks. Rats were euthanized 2 weeks after surgery and the left cochlear nuclei were harvested. The hearing level of each animal was estimated by using auditory brainstem responses (ABRs) before and after surgery and before euthanasia. Western blotting was performed using protein extracts obtained from the cochlear nuclei.

\section{Cochlear ablation}

Animals were anesthetized by intramuscular administration of Zoletil (40 mg/kg, Zoletil 50; Virbac, Bogotá, Colombia) mixed with xylazine $(10 \mathrm{mg} / \mathrm{kg}$, Rompun; Bayer-Korea, Seoul, Korea). After local anesthesia with $1 \%$ lidocaine hydrochloride, an incision was made at the left retroauricular site. The external auditory canal was identified and opened to expose the tympanic membrane. The tympanic membrane and ossicles were removed, except for the stapes. Then, the bony wall of the cochlea was identified and broken with a dental pick. The contents of the cochlea were also ablated with a dental pick. A small amount of soft tissue was harvested at the incision site and packed into the vacant cochlear space. The skin incision was closed with sutures. The right side was preserved without surgery. In the sham operation, the same operative procedure was performed up to the point of opening the external auditory canal.

\section{$A B R$ recordings}

ABRs were recorded with subdermal needle electrodes between the left mastoid and the nape of the neck with the right mastoid as the return. Tone pips of 8,16 , and $32 \mathrm{kHz}$ were used as sound stimuli (5-ms duration, cos shaping, $21 \mathrm{~Hz}$ ). ABRs were recorded with high-frequency transducers (HFT9911-20-0035) and software (ver. 2.33) from SmartEP (Intelligent Hearing Systems, Glenvar Heights, FL, USA). The responses were amplified $(100,000 \times)$, band pass-filtered $(100-1,500 \mathrm{~Hz})$ and averaged over 512 stimulus repetitions. Stimulus intensity was lowered in 5 -dB sound pressure level (SPL) decrements. The lowest stimulus intensity that evoked a recognizable response as determined 
by two researchers (SKM and $\mathrm{KHH}$ ) blinded to the experimental group was considered as the threshold.

\section{Western blot assay}

Animals were euthanized 1 or 2 weeks after surgery and the brain tissue was harvested. The location of the cochlear nucleus of the brainstem was identified according to the atlas of Paxinos and Watson [11] ( $-9.80 \mathrm{~mm}$ to $-11.30 \mathrm{~mm}$ from the bregma). The cochlear nucleus on the left side was dissected under a microscope, frozen in cryopreservation tubes in liquid nitrogen and stored at $-80^{\circ} \mathrm{C}$. The samples were homogenized in lysis buffer (Pro-Prep protein extraction solution; iNtRON Biotechnology, Seongnam, Korea) on ice. Homogenates were incubated on ice for at least 1 hour. After 1 hour, the extracts were centrifuged at $15,000 \mathrm{rpm}$ for 30 minutes at $4^{\circ} \mathrm{C}$ (Microcentrifuges, 5424R; Eppendorf, Hamburg, Germany). The protein concentration in the supernatant was measured with a Bradford assay and the proteins were denatured at $95^{\circ} \mathrm{C}$ for 5 minutes in sample buffer $(4 \times$, Laemmli SDS-sample buffer; GenDEPOT, Barker, TX, USA). Proteins were electrophoresed on an SDS-PAGE gel (WIDE One- Step Acryl Solution, \#CBWL-1000; Chembio, Medford, NY, USA). After electrophoresis, protein bands were transferred to PVDF membranes (Immune-Blot PVDF membrane for protein blotting; Bio-Rad, Hercules, CA, USA). The membranes were blocked with blocking solution ( $2 \%$ bovine serum albumin [BSA] in Tris buffered saline with Tween 20 [TBST]) overnight and then incubated overnight at $4^{\circ} \mathrm{C}$ with primary antibodies. In the first experiment, the membranes were probed with primary antibodies for VGLUT1 and VGLUT2. In the second experiment, primary antibodies for VGLUT1, VGLUT2, p-Smad2/3 and growth-associated protein 43 (GAP-43) were used. The primary antibodies were diluted in TBST containing $2 \%$ BSA using the following dilutions: rabbit $\beta$-actin, 1:2,000 (\#ab8227; Abcam, Cambridge, England); rabbit VGLUT1, 1:1,000 (\#ab77822, Abcam); mouse VGLUT2, 1:1,000 (\#ab79157, Abcam); rabbit p-Smad2/3, 1:1,000 (\#8828; Cell Signaling Technol- ogy, Danvers, MA, USA) and mouse GAP-43, 1:1,000 (\#MAB347; Millipore, Burlington, MA, USA). After several washes, membranes were incubated in a species-specific secondary antibody conjugated to horseradish peroxidase for 1 hour at room temperature. The secondary antibodies were diluted in TBST containing 2\% BSA (anti-rabbit: 1:2,000, \#5127S, Cell Signaling Technology; anti-mouse: 1:2,000, \#7076, Cell Signaling Technology). The chemiluminescence of the secondary antibodies was detected with enhanced chemiluminescence (ECL) detection (ECL Prime Western Blotting Detection Reagent; GE Healthcare, Little Chalfont, England) and analyzed with a chemiluminescence image analyzer system (FluorChem HD2; Cell Biosciences, Heidelberg, Germany).

\section{Statistical analysis}

Statistical analysis was carried out with IBM SPSS ver. 21.0 (IBM Corp., Armonk, NY, USA). The Kruskal-Wallis and Mann-Whitney $U$-tests were used. $P$-values less than 0.05 were considered statistically significant.

\section{RESULTS}

Time course of auditory-somatosensory plasticity following hearing loss

To identify the time point when the balance of auditory and somatosensory inputs was disrupted, cochlear nuclei of the brainstem were harvested 1 and 2 weeks after surgery.

\section{ABR recordings}

Baseline $A B R$ thresholds before surgery ranged from 20 to 35 dB SPL. ABR thresholds did not differ significantly between the groups before surgery $(P>0.05)$ (Fig. 1A). After surgery and before euthanasia, $A B R$ thresholds in the control group ranged from 20 to $35 \mathrm{~dB}$ SPL, while ABR thresholds in the deaf group were higher than $80 \mathrm{~dB}$ SPL (Fig. 1B).
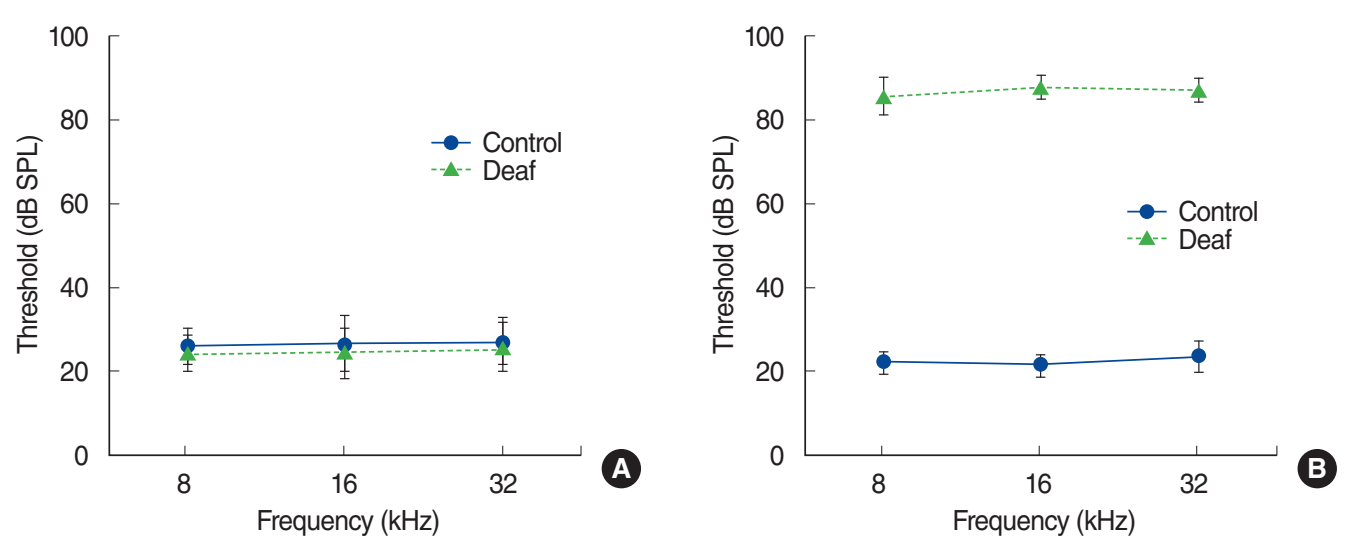

Fig. 1. Auditory brainstem response thresholds for the control and deaf groups before (A) and after (B) surgery. Error bars indicate standard deviation. SPL, sound pressure level. 

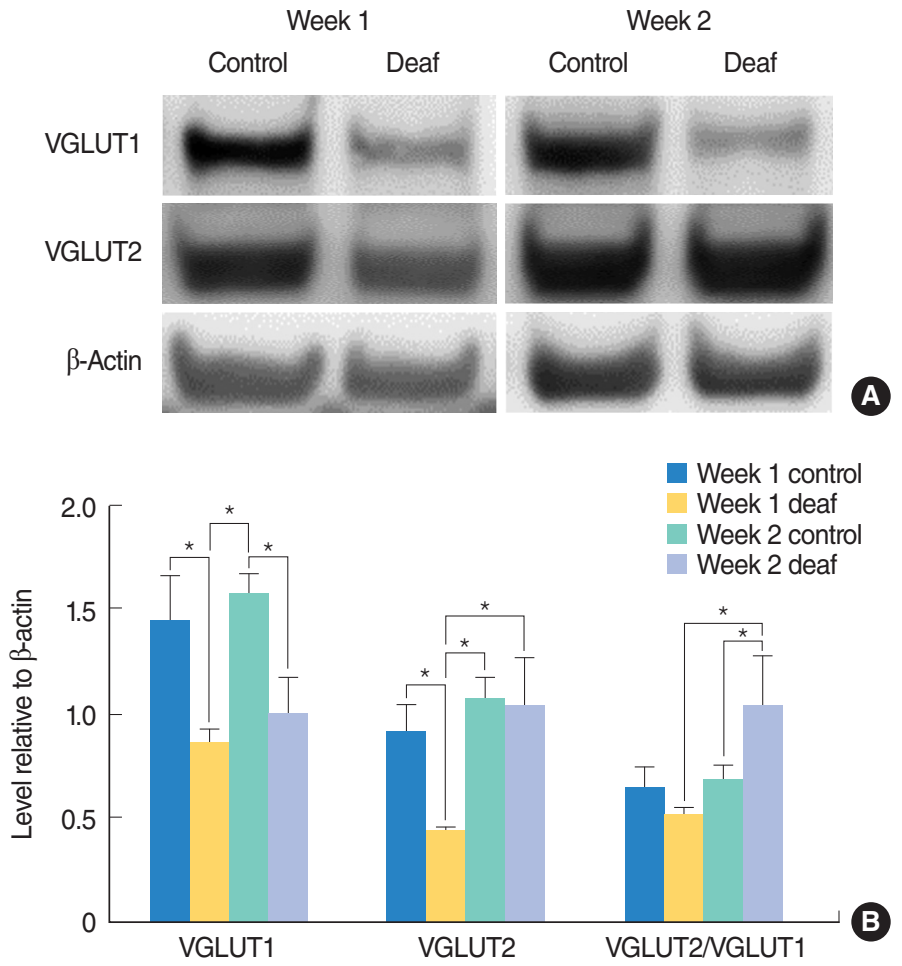

Fig. 2. Increase in the VGLUT2/VGLUT1 ratio after hearing loss VGLUT1 and VGLUT2 expression levels in the cochlear nucleus were examined with a Western blot assay 1 and 2 weeks after surgery. (A) Representative Western blots. (B) Quantitative analysis of VGLUT1 and VGLUT2. At 2 weeks after surgery, the deaf group had a significantly higher VGLUT2NGLUT1 ratio compared to the control group. Values are presented as mean \pm standard error. VGLUT1, vesicular glutamate transporter 1; VGLUT2, vesicular glutamate transporter 2. ${ }^{*}$ Statistically significant differences between groups, $P<0.05$.

\section{Disruption of the VGLUT2NGLUT1 ratio after hearing loss}

The expression levels of VGLUT1 were analyzed for the four groups: week 1 control, week 1 deaf, week 2 control, and week 2 deaf. There were significant differences among the four groups $(P=0.007)$ (Fig. 2). The week 1 deaf group had significantly lower levels of VGLUT1 compared to the week 1 and 2 control groups ( $P=0.035$ and $P=0.003$ ). The week 2 deaf group had significantly lower levels of VGLUT1 compared to the week 2 control group $(P=0.017)$. When changes were analyzed over time, there was no significant difference between the week 1 and 2 control groups and the week 1 and 2 deaf groups (Fig. 2). The expression levels of VGLUT2 were also analyzed in the same manner as the expression levels of VGLUT1. There were significant differences among the four groups $(P=0.007)$ (Fig. 2). The week 1 deaf group had significantly lower levels of VGLUT2 compared to the week 1 and 2 control groups ( $P=0.025$ and $P=0.003)$. However, the level of VGLUT2 in the week 2 deaf group was not significantly different compared to the week 1 and 2 control groups. When changes were analyzed over time, there was no significant difference in VGLUT2 levels between
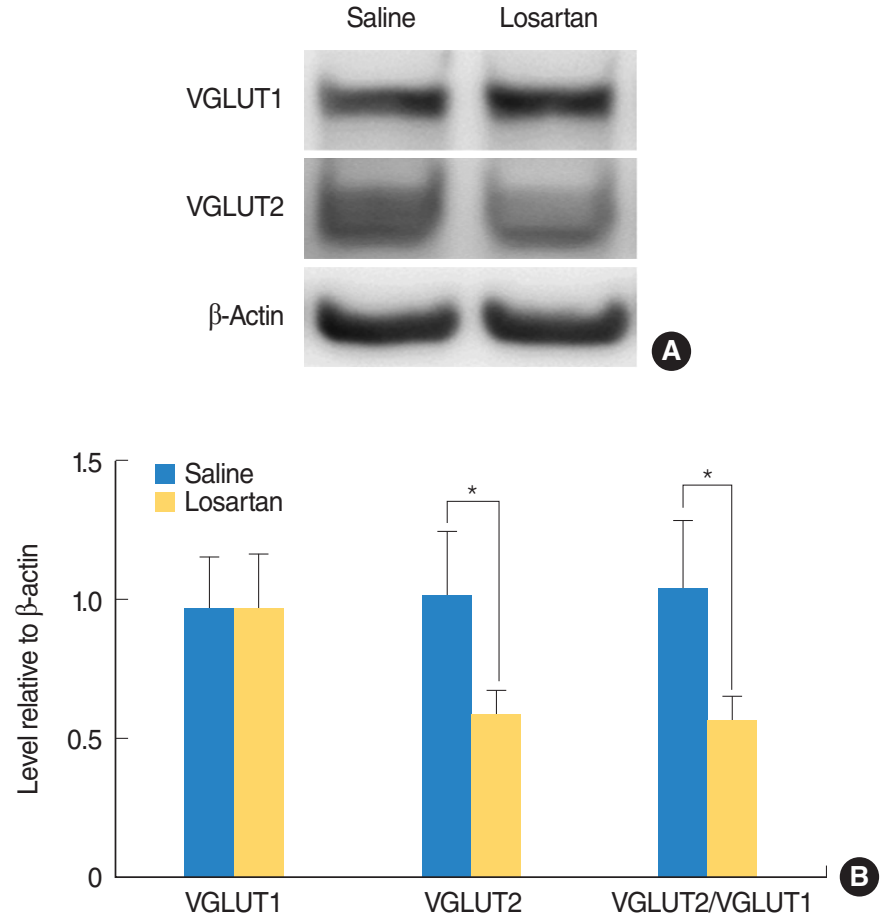

Fig. 3. Prevention of the increase in the VGLUT2NGLUT1 ratio using losartan. VGLUT1 and VGLUT2 expression levels in the cochlear nucleus were examined with a Western blot assay 2 weeks after surgery. (A) Representative Western blots. (B) Quantitative analysis of VGLUT1 and VGLUT2. The losartan group had a significantly lower VGLUT2NGLUT1 ratio compared to the deaf group. Values are presented as mean \pm standard error. VGLUT1, vesicular glutamate transporter 1; VGLUT2, vesicular glutamate transporter 2. *Statistically significant differences between groups, $P<0.05$.

the week 1 and 2 control groups. However, the week 2 deaf group had significantly higher levels of VGLUT2 compared to the week 1 deaf group ( $P=0.004)$ (Fig. 2).TheVGLUT2/VGLUT1 ratios were analyzed in the same manner as the expression levels of VGLUT1 and VGLUT 2. There were significant differences among the four groups $(P=0.039)$ (Fig. 2). The week 2 deaf group had a significantly higher VGLUT2/VGLUT1 ratio compared to the week 2 control group $(P=0.045)$. When changes were analyzed over time, the week 2 deaf group had a significantly higher VGLUT2/VGLUT1 ratio compared to the week 1 deaf group $(P=0.010)$ (Fig. 2).

Prevention of auditory-somatosensory plasticity using losartan Based on the results of the first experiment, the balance of auditory and somatosensory inputs was disrupted 2 weeks after hearing loss. We then investigated whether losartan prevented disruption of the VGLUT2/VGLUT1 ratio.

\section{ABR recordings}

Baseline ABR thresholds before surgery ranged from 20 to 35 
dB SPL. ABR thresholds did not differ significantly between the groups before surgery $(P>0.05)$. After surgery and before euthanasia, ABR thresholds in the saline and losartan groups were higher than $80 \mathrm{~dB}$ SPL.

\section{Prevention of disruption of the VGLUT2NGLUT1 ratio}

VGLUT1 levels in the cochlear nuclei did not differ significantly between the losartan and saline groups. VGLUT2 levels were significantly lower in the losartan group than in the saline group $(P=0.045)$ (Fig. 3). Additionally, the VGLUT2/VGLUT1 ratio was significantly lower in the losartan group than in the saline group $(P=0.028)$ (Fig. 3).

\section{p-Smad2/3 signaling and GAP-43}

p-Smad3 levels in the cochlear nuclei were significantly lower in the losartan group than in the saline group $(P=0.047)$ (Fig. 4). pSmad2 levels had a pattern similar to p-Smad3, but the results were not statistically significant. GAP-43 levels were significantly lower in the losartan group than in the saline group $(P=0.045)$ (Fig. 4).
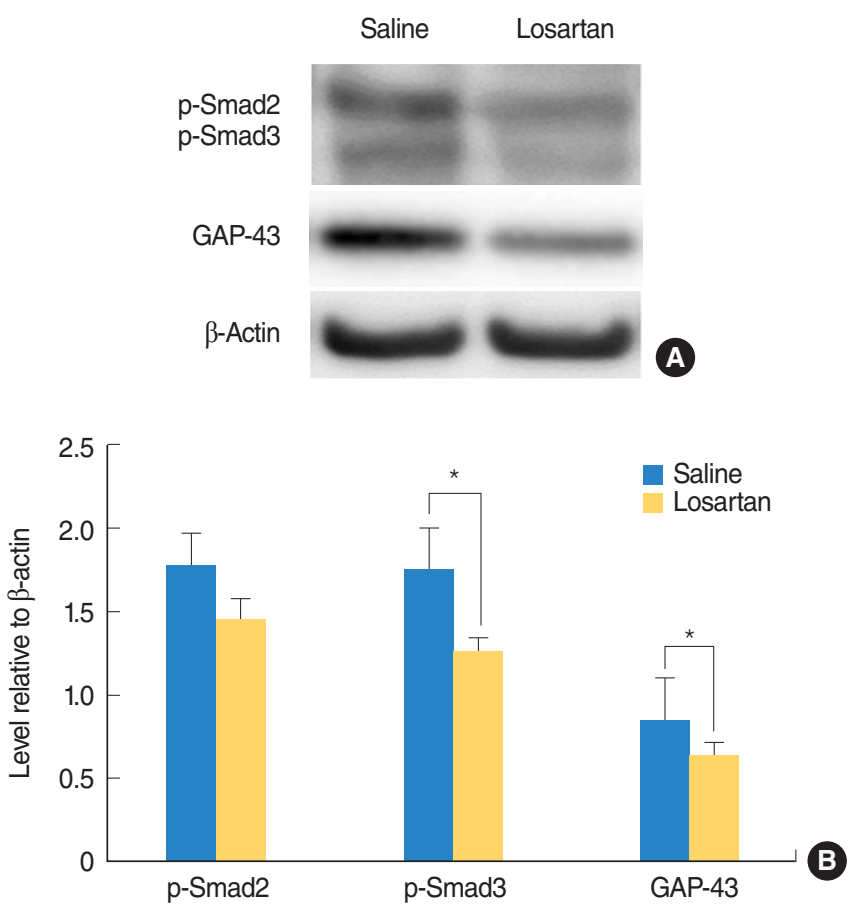

Fig. 4. Changes in p-Smad2 and 3 signaling, and GAP-43 following losartan administration. p-Smad2 and 3, and GAP-43 expression levels in the cochlear nucleus were examined with a Western blot assay 2 weeks after surgery. (A) Representative Western blots. (B) Quantitative analysis of p-Smad2 and 3, and GAP-43. The losartan group had significantly lower p-Smad3 and GAP-43 levels compared to the deaf group. Values are presented as mean \pm standard error. GAP-43, growth-associated protein 43. *Statistically significant differences between groups, $P<0.05$.

\section{DISCUSSION}

The disruption of the balance of auditory and somatosensory inputs, which occurs in the cochlear nucleus after hearing loss, was first suggested in 2009. Zeng et al. [6] showed that VGLUT1 expression decreased and VGLUT2 expression increased in the cochlear nucleus after hearing loss. Based on their previous findings that VGLUT1 and VGLUT2 are differentially associated with auditory and somatosensory inputs to the cochlear nucleus, respectively [8], they suggested that hearing loss causes a decrease in auditory inputs and compensatory increase in somatosensory inputs in the cochlear nucleus, leading to a disrupted balance of auditory and somatosensory inputs. This potential mechanism was confirmed through VGLUT immunohistochemistry combined with anterograde tract tracing, which showed that higher levels of VGLUT2 expression are representative of an increase in projections from the somatosensory pathways to the cochlear nucleus [7]. Subsequently, several studies have shown that a disrupted balance of auditory and somatosensory inputs causes aberrant neural activity in the cochlear nucleus, contributing to the development of tinnitus $[1,4,5,7,12]$. Therefore, potential therapies for tinnitus may be found in preventing the disruption of the balance between auditory and somatosensory inputs in the cochlear nucleus after hearing loss.

The disruption in the balance of auditory-somatosensory inputs following hearing loss is thought to be due to axonal sprouting through maladaptive auditory-somatosensory plasticity [6]. Several studies have reported evidence that axonal sprouting occurs in the cochlear nucleus after hearing loss, such as the reemergence of GAP-43 and synaptophysin [13-15]. However, to date there has been no attempt to prevent the disruption of the balance between auditory and somatosensory inputs in the cochlear nucleus. In this study, we attempted to prevent axonal sprouting and to avoid the disruption of the balance between auditory and somatosensory inputs in the cochlear nucleus after hearing loss for the first time. We hypothesized that blocking axonal sprouting would prevent an increase in the VGLUT2/ VGLUT1 ratio after hearing loss. First, we identified the time point when the VGLUT2/VGLUT1 ratio increased after hearing loss. Then, we investigated whether losartan prevented the increase in the VGLUT2/VGLUT1 ratio. Systemic losartan injection has been reported to inhibitTGF- $\beta$ signaling both in peripheral tissue [16] and the brain $[17,18]$. Because TGF- $\beta$ signaling promotes axonal sprouting in the brain [18], we attempted to block axonal sprouting by inhibiting TGF- $\beta$ signaling with losar$\tan$. As p-Smad $2 / 3$ is downstream of TGF $\beta$ receptors, we measured $\mathrm{p}$-Smad2/3 levels to demonstrate that losartan affected TGF- $\beta$ signaling $[17,18]$. The levels of GAP-43, a well-established marker for synaptogenesis and axonal outgrowth [19], were measured to assess axonal sprouting.

In the first experiment, we found that VGLUT1 levels decreased 1 week after hearing loss, as previously reported [6,7]. 
This trend continued at 2 weeks after hearing loss. However, VGLUT2 levels decreased at 1 week after hearing loss and increased at 2 weeks after hearing loss. There has been no report showing a decrease in VGLUT2 levels at 1 week after hearing loss to date. This phenomenon may be due to the degeneration of the neurons receiving both auditory and somatosensory inputs. These neurons may degenerate as a consequence of reduced afferent-derived neurotrophic support from auditory inputs [14], which may lead to a decrease in the somatosensory projection to the cochlear nucleus. Further studies are needed to confirm the mechanism of this phenomenon. VGLUT2 levels at 2 weeks after hearing loss increased more than at 1 week after hearing loss. Consequently, the VGLUT2/VGLUT1 ratio increased at 2 weeks after hearing loss. This result is consistent with previous studies [6]. In the second experiment, we found that VGLUT2 levels in the losartan group were significantly lower than in the saline group 2 weeks after hearing loss. Losartan administration might prevent the generation of new VGLUT2 after hearing loss. In other words, losartan administration might prevent an increase in somatosensory inputs by blocking axonal sprouting. This hypothesis was supported by a decrease in GAP43 in the losartan group. The losartan group also had lower pSmad2/3 levels compared to the deaf group. This result provides evidence that losartan inhibited TGF- $\beta$ activation after hearing loss. Losartan also prevented the increase in the VGLUT2/ VGLUT1 ratio in the cochlear nucleus after hearing loss and maintained the balance of auditory and somatosensory inputs after hearing loss.

The present study has a few limitations. Most importantly, we investigated the effect of losartan in a hearing loss model, not a tinnitus model, so the results may not be applicable to tinnitus. However, because hearing loss is the most important causative factor of tinnitus, the results of this study can provide clues to mechanisms of and potential therapies for tinnitus. In addition, this is the first study that has prevented maladaptive auditorysomatosensory plasticity in the cochlear nucleus after hearing loss. Based on this result, future studies will be conducted to prevent changes in the central auditory pathway caused by hearing loss.

In summary, we suggest that losartan might prevent axonal sprouting, which takes place in the cochlear nucleus after hearing loss, by blocking TGF- $\beta$ signaling, thereby preventing disruption of the balance of auditory and somatosensory inputs.

\section{CONFLICT OF INTEREST}

No potential conflict of interest relevant to this article was reported.

\section{ACKNOWLEDGMENTS}

This work was supported by a National Research Foundation of Korea (NRF) grant funded by the Korea Government (Ministry of Science, ICT and Future Planning) (No. 2016R1C1B2007131 to MYC), a research grant from Biomedical Research Institute, Chung-Ang University Hospital (2017 to MYC), the Public Health Research Program funded by National Medical Center, Research Institute (No. NMC2017-PR-00 to KHH) and Otolaryngology Alumni Association of Chung-Ang University Hospital (to SKM).

\section{REFERENCES}

1. Wu C, Stefanescu RA, Martel DT, Shore SE. Tinnitus: maladaptive auditory-somatosensory plasticity. Hear Res. 2016 Apr;334:20-9.

2. Zhan X, Pongstaporn T, Ryugo DK. Projections of the second cervical dorsal root ganglion to the cochlear nucleus in rats. J Comp Neurol. 2006 May;496(3):335-48.

3. Zhou J, Shore S. Convergence of spinal trigeminal and cochlear nucleus projections in the inferior colliculus of the guinea pig. J Comp Neurol. 2006 Mar;495(1):100-12.

4. Dehmel S, Pradhan S, Koehler S, Bledsoe S, Shore S. Noise overexposure alters long-term somatosensory-auditory processing in the dorsal cochlear nucleus: possible basis for tinnitus-related hyperactivity? J Neurosci. 2012 Feb;32(5):1660-71.

5. Koehler SD, Shore SE. Stimulus timing-dependent plasticity in dorsal cochlear nucleus is altered in tinnitus. J Neurosci. 2013 Dec;33(50): 19647-56

6. Zeng C, Nannapaneni N, Zhou J, Hughes LF, Shore S. Cochlear damage changes the distribution of vesicular glutamate transporters associated with auditory and nonauditory inputs to the cochlear nucleus. J Neurosci. 2009 Apr;29(13):4210-7.

7. Zeng C, Yang Z, Shreve L, Bledsoe S, Shore S. Somatosensory projections to cochlear nucleus are upregulated after unilateral deafness. J Neurosci. 2012 Nov;32(45):15791-801.

8. Zhou J, Nannapaneni N, Shore S. Vessicular glutamate transporters 1 and 2 are differentially associated with auditory nerve and spinal trigeminal inputs to the cochlear nucleus. J Comp Neurol. 2007 Feb; 500(4):777-87.

9. Zeng C, Shroff H, Shore SE. Cuneate and spinal trigeminal nucleus projections to the cochlear nucleus are differentially associated with vesicular glutamate transporter-2. Neuroscience. 2011 Mar;176:14251.

10. Boulland JL, Ferhat L, Tallak Solbu T, Ferrand N, Chaudhry FA, Storm-Mathisen J, et al. Changes in vesicular transporters for gamma-aminobutyric acid and glutamate reveal vulnerability and reorganization of hippocampal neurons following pilocarpine-induced seizures. J Comp Neurol. 2007 Jul;503(3):466-85.

11. Paxinos G, Watson C. The rat brain in stereotaxic coordinates. 6th ed. Burlington (MA): Academic Press; 2006.

12. Koehler SD, Shore SE. Stimulus-timing dependent multisensory plasticity in the guinea pig dorsal cochlear nucleus. PLoS One. 2013;8(3): e59828.

13. Bilak M, Kim J, Potashner SJ, Bohne BA, Morest DK. New growth of axons in the cochlear nucleus of adult chinchillas after acoustic trauma. Exp Neurol. 1997 Oct;147(2):256-68.

14. Fuentes-Santamaria V, Alvarado JC, Henkel CK, Brunso-Bechtold JK. Cochlear ablation in adult ferrets results in changes in insulin- 
like growth factor-1 and synaptophysin immunostaining in the cochlear nucleus. Neuroscience. 2007 Sep;148(4):1033-47.

15. Illing RB, Horvath M. Re-emergence of GAP-43 in cochlear nucleus and superior olive following cochlear ablation in the rat. Neurosci Lett. 1995 Jul;194(1-2):9-12.

16. Cohn RD, van Erp C, Habashi JP, Soleimani AA, Klein EC, Lisi MT, et al. Angiotensin II type 1 receptor blockade attenuates TGF-betainduced failure of muscle regeneration in multiple myopathic states. Nat Med. 2007 Feb;13(2):204-10.
17. Bar-Klein G, Cacheaux LP, Kamintsky L, Prager O, Weissberg I, Schoknecht K, et al. Losartan prevents acquired epilepsy via TGF- $\beta$ signaling suppression. Ann Neurol. 2014 Jun;75(6):864-75.

18. Li S, Nie EH, Yin Y, Benowitz LI, Tung S, Vinters HV, et al. GDF10 is a signal for axonal sprouting and functional recovery after stroke. Nat Neurosci. 2015 Dec;18(12):1737-45.

19. Benowitz LI, Routtenberg A. GAP-43: an intrinsic determinant of neuronal development and plasticity. Trends Neurosci. 1997 Feb; 20(2):84-91. 